\title{
Article \\ Improvement of Mechanical Properties with Non-Equimolar CrNbTaVW High Entropy Alloy
}

\author{
Francisco Antão ${ }^{1}$, Ricardo Martins ${ }^{1}$, José Brito Correia ${ }^{2}$, Rui Coelho da Silva ${ }^{1} \mathbb{D}$, António Pereira Gonçalves ${ }^{3} \mathbb{D}$, \\ Elena Tejado ${ }^{4}\left(\mathbb{D}\right.$, José Ygnacio Pastor ${ }^{4}\left(\mathbb{D}\right.$, Eduardo Alves ${ }^{1}(\mathbb{D})$ and Marta Dias ${ }^{1, *}$
}

1 Instituto de Plasmas e Fusão Nuclear, Instituto Superior Técnico, Universidade de Lisboa, Av. Rovisco Pais, 1049-001 Lisboa, Portugal; fantao@ctn.tecnico.ulisboa.pt (F.A.); ricardo.martins@ctn.tecnico.ulisboa.pt (R.M.); rmcs@ctn.tecnico.ulisboa.pt (R.C.d.S.); ealves@ctn.tecnico.ulisboa.pt (E.A.)

2 LNEG, Laboratório Nacional de Energia e Geologia, Estrada do Paço do Lumiar, 1649-038 Lisboa, Portugal; brito.correia@lneg.pt

3 C2TN, Instituto Superior Técnico, Universidade de Lisboa, Campus Tecnológico e Nuclear, Estrada Nacional 10, 2695-066 Bobadela, Portugal; apg@ctn.tecnico.ulisboa.pt

4 Departamento de Ciencia de Materiales-CIME, ETSI Caminos, Canales y Puertos, Universidad Politécnica de Madrid, 28040 Madrid, Spain; elena.tejado@upm.es (E.T.); jy.pastor@upm.es (J.Y.P.)

* Correspondence: marta.dias@ctn.ist.utl.pt; Tel.: +351-219946000; Fax: +351-219550117

\section{check for}

updates

Citation: Antão, F.; Martins, R.;

Correia, J.B.; da Silva, R.C.;

Gonçalves, A.P.; Tejado, E.; Pastor,

J.Y.; Alves, E.; Dias, M. Improvement

of Mechanical Properties with

Non-Equimolar CrNbTaVW High

Entropy Alloy. Crystals 2022, 12, 219.

https://doi.org/10.3390/

cryst12020219

Academic Editors: Rui Feng, Ke An and Peter K. Liaw

Received: 17 January 2022

Accepted: 28 January 2022

Published: 1 February 2022

Publisher's Note: MDPI stays neutral with regard to jurisdictional claims in published maps and institutional affiliations.

Copyright: (C) 2022 by the authors. Licensee MDPI, Basel, Switzerland. This article is an open access article distributed under the terms and conditions of the Creative Commons Attribution (CC BY) license (https:// creativecommons.org/licenses/by/ $4.0 /)$

\begin{abstract}
CrNbTaVW}_{\mathrm{x}}$ with ( $\mathrm{x}=1$ and 1.7) high entropy alloys have been devised for thermal barriers between the plasma-facing tungsten tiles and the copper-based heat sink in the first wall of fusion nuclear reactors. These novel materials were prepared by ball milling and consolidated by Upgrade Field Assisted Sintering Technology at $1873 \mathrm{~K}$ under an applied pressure of $90 \mathrm{MPa}$ for $10 \mathrm{~min}$. In this work, the structural and mechanical properties of these materials were evaluated. Consolidated samples presented a major phase with a bcc-type structure with lattice parameter value of $0.316 \mathrm{~nm}$ for $\mathrm{CrNbTaVW}_{\text {and }} \mathrm{CrNbTaVW}_{1.7}$ compositions. Moreover, observation of the microstructures evidences also two minor phases: Ta-Nb-Cr and Ta- $\mathrm{V}$ rich (in which carbon is detected). Despite the similarity in the structural properties of these two alloys, their mechanical properties are distinct. The flexural stress for the sample with higher amount of $\mathrm{W}\left(\mathrm{CrNbTaVW}_{1.7}\right)$ is higher by $50 \%$ in the $298-873 \mathrm{~K}$ range, with an increased strain to fracture, which can be associated with reduced brittleness caused by the additional $\mathrm{W}$ incorporation.
\end{abstract}

Keywords: high entropy alloys; mechanical alloying; consolidation; microstructure; mechanical properties

\section{Introduction}

Tungsten is considered the best candidate for plasma facing tiles due to its endurance for high temperature and irradiation resilience and resistance, high melting point, low sputtering rate, and low tritium retention [1]. However, the currently available grades are brittle at temperatures below $623 \mathrm{~K}$, due to the occurrence of a ductile-to-brittle transition in the range of $623 \mathrm{~K}$ to $873 \mathrm{~K}$ [2]. On the other hand, the material selected for heat sink behind the plasma-facing components is a $\mathrm{CuCrZr}$ alloy with a service temperature limited to $\sim 623 \mathrm{~K}$ [3]. These two materials, $\mathrm{W}$ and $\mathrm{CuCrZr}$ alloy, thus show a large mismatch in working temperatures, demanding resourcefulness solutions for their joint operation. A possible solution is the introduction of a thermal barrier interlayer between these two components. One approach to solve this issue is the use of high entropy alloys compatible with both $\mathrm{W}$ and the $\mathrm{CuCrZr}$ alloys which can work as a thermal barrier interlayer capable of minimizing the thermal mismatch between both materials, keeping them in their required work temperature intervals.

High-entropy alloys (HEAs) are a class of materials developed in recent years which exhibit excellent properties for this application, such as high hardness [4], high thermal 
stability [5], and low thermal diffusivity [5,6]. In general, HEAs usually possess unique microstructures and, consequently, different properties [7-10] that can be tuned according to the task envisaged. The use of HEAs based on refractory-elements $(\mathrm{Mo}, \mathrm{Nb}, \mathrm{Ta}, \mathrm{W}$, and V) was first introduced by Senkov et al. [11] mainly as candidates for structural applications at temperatures above $1373 \mathrm{~K}$, but subsequent alloys have been drawn from a broader palette of nine elements in Group IV ( $\mathrm{Ti}, \mathrm{Zr}$, and $\mathrm{Hf}$ ), Group V (V, Nb, and $\mathrm{Ta}$ ), and Group VI (Cr, Mo, and W). Many refractory HEAs were subsequently developed to obtain improved strength, room temperature ductility, and high hardness up to $1873 \mathrm{~K}$. Despite the numerous studies on refractory HEAs, only a few recent works have been devoted to the high temperature class of alloys, with output on microstructure characterization and mechanical properties $[10,11]$. This research presents a study of the $\mathrm{CrNbTaVW}_{\mathrm{x}}$ high entropy alloys changing the $W$ amount $(x=1$ and 1.7), including its synthesis method and resulting microstructure together with its high temperature mechanical properties. Therefore, the study of the effect of the increasing $\mathrm{W}$ content, on the equiatomic $\mathrm{CrNbTaVW}$ in terms of structural and mechanical properties, is the goal of the present work.

\section{Experimentals}

The $\mathrm{W}, \mathrm{Cr}, \mathrm{Ta}, \mathrm{Nb}$, and $\mathrm{V}$ powders (AlfaAesar, nominal purity of $99.9 \%$ with average particle size of $10 \mu \mathrm{m}$ ) were mixed in two different proportions: equiatomic and nonequiatomic in a glove box and mechanically alloyed in a high-energy planetary ball mill, PM 400 MA, with WC balls and vials. The balls to powder mass ratio was 10:1, and the milling was carried out at $380 \mathrm{rpm}$ for effective times up to $2 \mathrm{~h}$. The samples and their respective designation are showed in Table 1.

Table 1. Atomic percentage of each element in all samples and the respective designation.

\begin{tabular}{ccccccc}
\hline $\begin{array}{c}\text { Samples } \\
\text { CrNbTaVW }_{\mathbf{x}}\end{array}$ & Designation & $\mathrm{Cr}$ (at.\%) & Nb (at.\%) & Ta (at.\%) & V (at.\%) & W (at.\%) \\
\hline $\begin{array}{c}\mathrm{x}=1 \\
\text { equiatomic }\end{array}$ & $\mathrm{CrNbTaVW}$ & 20 & 20 & 20 & 20 & 20 \\
\hline $\begin{array}{c}\mathrm{x}=1.7 \\
\text { non-equiatomic }\end{array}$ & $\mathrm{CrNbTaVW}_{1.7}$ & 17.5 & 17.5 & 17.5 & 17.5 & 30 \\
\hline
\end{tabular}

The consolidation was performed in graphite dies of $10 \mathrm{~mm}$ diameter and $5 \mathrm{~mm}$ height by the Upgraded Field Assisted Sintering technology (U-FAST) process. A preliminary degassing step was conducted at $873 \mathrm{~K}$ for $2 \mathrm{~min}$ under a pressure of $15 \mathrm{MPa}$. The samples were then sealed under vacuum $\left(5 \times 10^{-3} \mathrm{~Pa}\right)$, at a temperature of $1873 \mathrm{~K}$ and an external hydrostatic pressure of $90 \mathrm{MPa}$ was applied for $10 \mathrm{~min}$ and then allowed to cool to room temperature. The final discs produced have a volume of $0.63 \mathrm{~cm}^{3}$ with an average weight of $6.86 \mathrm{~g}$. After sintering, the apparent density was measured by the Archimedes method.

Powder X-ray diffraction (PXRD) was used to investigate the evolution of the powders mixtures before and after the $2 \mathrm{~h}$ milling. Measurements were made using a Philips X'Pert diffractometer in a Bragg-Brentano geometry with $\mathrm{Cu} \mathrm{K}$-alpha radiation, over a 2 range from $10^{\circ}$ to $100^{\circ}$ with a 2 step size of $0.03^{\circ}$. The consolidated samples were studied using a Bruker D8 AXS diffractometer in a grazing incidence geometry (GIXRD), with Cu K radiation incident at an angle of $3^{\circ}$, over 2 range from $20^{\circ}$ to $90^{\circ}$ with a $2 \theta$ step size of $0.02^{\circ}$. The ICDD database [12] was used for phase identification. The Powder Cell software package [13] was employed to simulate diffractograms for comparison with experimental data.

The metallographic preparation involved grinding with $\mathrm{SiC}$ paper and polishing with $6 \mu \mathrm{m}, 3 \mu \mathrm{m}$, and $1 \mu \mathrm{m}$ grade diamond suspensions. The microstructures were later observed in backscattered electron imaging (BSE) and secondary electron imaging (SE) modes using a JEOL JSM-7001F field emission gun scanning electron microscope (SEM) equipped with an Oxford Instruments X-ray EDS system. Quantitative analyses were 
performed by energy dispersive spectrometry (EDS) of the X-rays emitted from the major phases present in the alloys. Each phase was analyzed in more than 10 randomly selected points. The EDS analyses were made in regions where the phases present extend over large areas in order to decrease the probability of spectral contamination or interference.

Thermodynamics calculations were performed to determine the possible structures formed in the sample, and to help with the interpretation and discussion of the results. Based on empirical models $[8,14]$ using the enthalpies and entropies of mixing, $\Delta H_{m i x}$ and $\Delta S_{m i x}$, the fractional atomic size differences $\delta$ and the valence electron concentrations, VEC, it is possible to predict the formation of solid solutions in the ranges $-15 \mathrm{~kJ} / \mathrm{mol} \leq \Delta H_{\text {mix }} \leq 5 \mathrm{~kJ} / \mathrm{mol}, 0.01 \leq \delta \leq 0.06$, and $11 \mathrm{~J} /(\mathrm{K} \cdot \mathrm{mol}) \leq \Delta S_{\text {mix }} \leq 19.5 \mathrm{~J} /(\mathrm{K} \cdot \mathrm{mol}):$ the stable most phases are predicted to be fcc-type structure at VEC $\geq 8$ and bcc-type structure at VEC $<6.87$. Between these values, mixed fcc and bcc-type structures are expected to coexist. In this context, the calculations of the relevant properties of the $\mathrm{CrNbTaVW}$ and $\mathrm{CrNbTaVW}_{1.7}$ high entropy alloys are presented in Table 2. Based on the calculated values, a predominance of bcc-type solid solutions is expected for both compositions.

Table 2. Thermodynamic calculations for the $\mathrm{CrNbTaVW}$ and $\mathrm{CrNbTaVW}_{1.7}$ high entropy alloys.

\begin{tabular}{ccccc}
\hline Composition & $\boldsymbol{\Delta}_{\boldsymbol{m i x}} \mathbf{~} \mathbf{J} / \mathbf{m o l}$ & $\left.\boldsymbol{\Delta} \boldsymbol{S}_{\text {mix }} \mathbf{J} / \mathbf{( K} \cdot \mathbf{m o l}\right)$ & $\boldsymbol{\delta} \times \mathbf{1 0 0}$ & VEC \\
\hline $\mathrm{CrNbTaVW}$ & -5.28 & 13.38 & 5.09 & 5.4 \\
$\mathrm{CrNbTaVW}_{1.7}$ & -5.35 & 13.15 & 4.48 & 5.48 \\
\hline
\end{tabular}

Vickers microhardness was obtained by applying a load of $9.8 \mathrm{~N}$ for $15 \mathrm{~s}$ on an AKASHI MVK-EIII tester. Additionally, nanoindentation to a depth of $1500 \mathrm{~nm}$ was performed on the same samples using a standard Berkovich tip, calibrated using fused silica. The hardness measurements were performed on random regions. The average values of hardness and elastic modulus were taken from the unloading curve according to the Oliver and Pharr method [15]. Lastly, smooth bars of $0.8 \times 2 \mathrm{~mm}^{2}$ sections were then cut by electro discharge machining for further mechanical testing. Quasi-static three-point bending (TPB) tests were then performed using $16 \mathrm{~mm}$ loading span at a constant cross head travel speed of $100 \mu \mathrm{m} / \mathrm{min}$ for easy control of the test process while obtaining accurate recording data. To investigate the flexural strength of these novel materials in their expected operating temperature range, TPB tests were performed at $298 \mathrm{~K}, 673 \mathrm{~K}$, and $873 \mathrm{~K}$ under a high vacuum atmosphere $\left(10^{-6} \mathrm{mbar}\right)$. The flexural strength was later computed using Euler-Bernoulli equations for slender beams up to failure and their fracture surfaces were evaluated.

\section{Results and Discussion}

Figure 1a shows the X-ray pattern of the evolution of the CrNbTaVW alloy in three stages of synthesis: powder mixture of raw elements before milling, the as-milled powder (after $2 \mathrm{~h}$ milling), and the sintered sample. Comparing the diffractograms presented in (a) it is possible to observe that after $2 \mathrm{~h}$ of milling, the diffraction peaks of the individual elements can no longer be observed, and a set of broad peaks has developed. Moreover, weak peaks assigned to WC, are present in the diffractogram of the milled powder, which are attributed to the use of WC balls and vials as milling media. On the other hand, the diffractogram of the sintered sample evidences sharp peaks, which in agreement with Figure $1 \mathrm{~b}$ correspond to a bcc-type structure with a lattice parameter $0.316 \mathrm{~nm}$ (observed already after $2 \mathrm{~h}$ milling as mentioned above) and another, minor phase (identified with the symbol * in Figure 1a with a lattice parameter of $0.334 \mathrm{~nm}$ ) and the presence of small non-identified peaks. Note that the diffractogram of the sintered sample did not reveal the presence of WC as was observed in the milled powder. The experimental diffratogram of the $\mathrm{CrNbTaVW}_{1.7}$ sample (Figure $1 \mathrm{~b}$ ) is very similar to the one presented for the equiatomic $\mathrm{CrNbTaVW}$ sample, with a major bcc-type structure phase with the same lattice parameter 
of $0.316 \mathrm{~nm}$. Due to the similarity of the two samples only the X-ray evolution for phase formation was shown for the equiatomic sample.

(a)

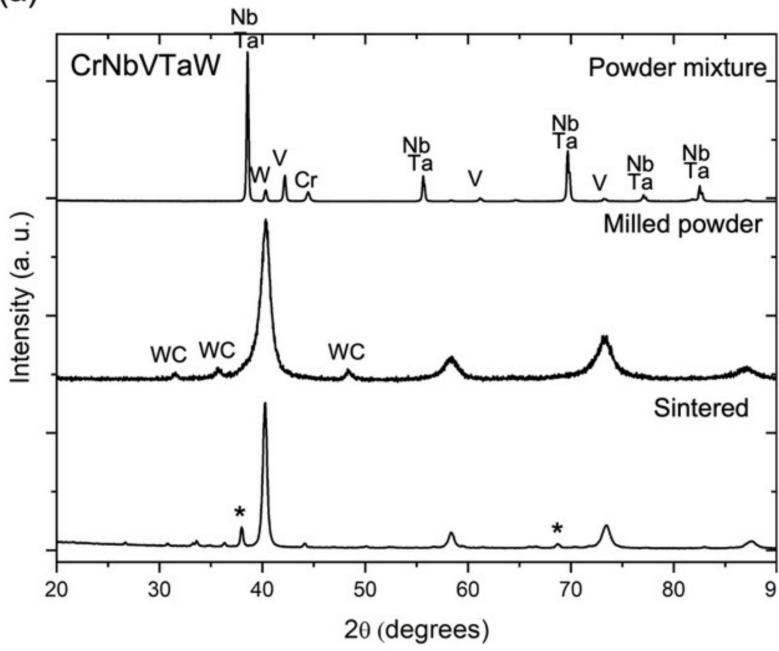

(b)

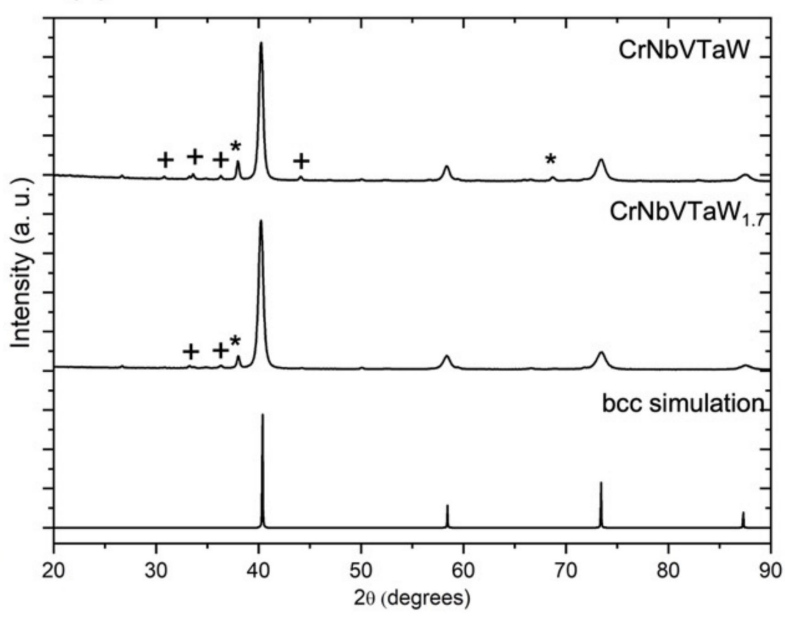

Figure 1. X-ray diffractogram of (a) evolution of the phase formation on $\mathrm{CrNbTaVW}$ high entropy alloy (powder mixture, milled powder and sintered sample) and (b) sintered CrNbTaVW and $\mathrm{CrNbTaVW}_{1.7}$ together with theoretical simulation of the bcc-type structure with $\mathrm{a}=0.316 \mathrm{~nm}$. The symbol * indicates the presence of a bcc-type structure theoretical simulation with $\mathrm{a}=0.334 \mathrm{~nm}$. The symbols + indicate minor unidentified structures.

Figure 2 displays only the milled powder of $\mathrm{CrNbTaVW}$ (since the $\mathrm{CrNbTaVW}_{1.7}$ powder is similar) and the microstructures of the sintered $\mathrm{CrNbTaVW}$ and $\mathrm{CrNbTaVW}_{1.7}$ high entropy alloys. The milled powder of the CrNbTaVW sample exhibits a particle distribution with a size less than 10 microns and revealed the mixture of the elements. The microstructures of the sintered samples are similar and no extensive porosity (less than $5 \%$ ) was observed in both samples, which is in agreement with the measured apparent density measured of $97 \%$ for $\mathrm{CrNbTaVW}$ and $95 \%$ for $\mathrm{CrNbTaVW}_{1.7}$. Both microstructures revealed the presence of a major phase (indicated as a white arrow in Figure $2 b, c$ ) and two uniformly dispersed dark phases, located in the boundaries of the submicron matrix grains (indicated as a black arrow in Figure $2 b, c)$. The EDS for the major phase for both compositions is evidenced in Table 3. The major phase for the equiatomic $\mathrm{CrNbTaVW}$ has a near equiatomic composition, however with less chromium and niobium while for the non-equiatomic $\mathrm{CrNbTaVW}_{1.7}$ exhibits a near initial composition, however with more chromium and less niobium. Moreover, the grain size of the major phase is around $2 \mu \mathrm{m}$ while for the dark phases is less than $1 \mu \mathrm{m}$. In fact, the contribution of the dark phases is minor compared to the major phase.
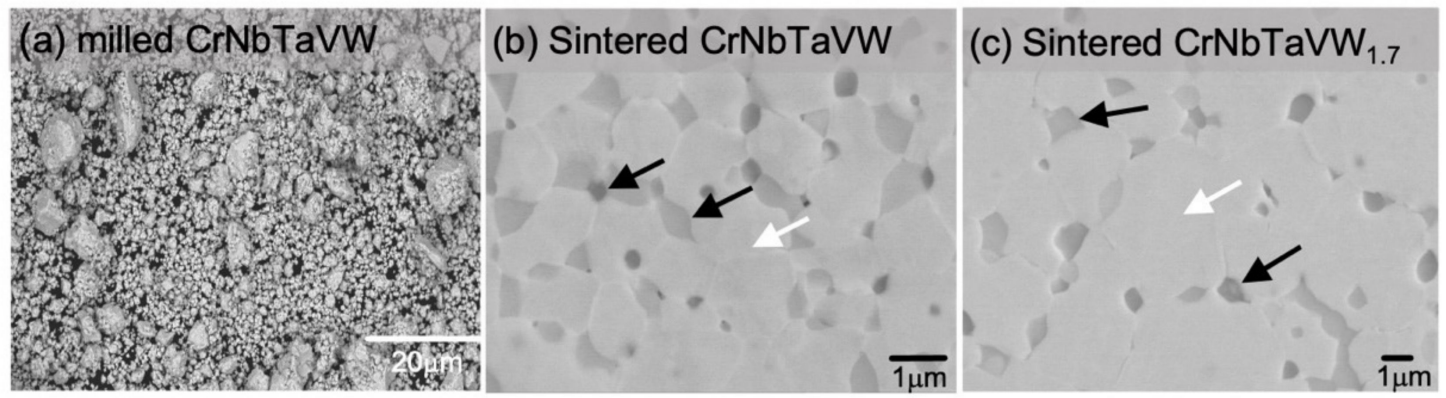

Figure 2. (a) BSE image of the milled powder of CrNbTaVW composition and microstructures of sintered (b) $\mathrm{CrNbTaVW}$ and (c) $\mathrm{CrNbTaVW}_{1.7}$ high entropy alloys. The white arrows in (b,c) indicate the presence of the major phase. The black arrows in $(\mathbf{b}, \mathbf{c})$ indicate the presence of the dark phases. 
Table 3. Average elemental composition on the major phase obtained by EDS analysis for both CrNbTaVW and $\mathrm{CrNbTaVW}_{1.7}$ compositions.

\begin{tabular}{cccccccc}
\hline Composition & & W at. $\%$ & Ta at. $\%$ & Cr at. $\%$ & Nb at. $\%$ & V at. $\%$ \\
\hline \multirow{2}{*}{ CrNbTaVW } & EDS & $23.52 \pm 0.59$ & $19.19 \pm 0.76$ & $20.42 \pm 0.68$ & $15.90 \pm 0.47$ & $20.97 \pm 0.40$ \\
\cline { 2 - 7 } & Nominal & 20 & 20 & 20 & 20 & 20 \\
\hline \multirow{2}{*}{ CrNbTaVW $_{1.7}$} & EDS & $30.59 \pm 0.32$ & $16.56 \pm 0.27$ & $19.88 \pm 0.32$ & $14.26 \pm 0.14$ & $18.70 \pm 0.18$ \\
\cline { 2 - 7 } & Nominal & 30 & 17.5 & 17.5 & 17.5 & 17.5 \\
\hline
\end{tabular}

Figure 3a-f shows the EDS map of the regions found on the microstructure of the sintered $\mathrm{CrNbTaVW}$ high entropy alloy. The microstructure shows the presence of a major phase, as discussed above, and two darker regions (which indicate that they are richer in light elements) which correspond to a Ta-Nb-Cr and Ta-V richer. Since regions Ta- $\mathrm{V}$ rich are darker when compared to the other phases, an EDS spectrum at low energy of this region is shown in Figure $3 \mathrm{~g}$ in order to distinguish light elements. In fact, the spectrum revealed a high intensity peak of $\mathrm{C}$ and $\mathrm{O}$, which can explain the lower atomic number of this phase and consequently the presence of a dark phase. This result is in agreement with what was observed in the XRD between the diffractogram of the milled powder and the sintered sample; the WC was only observed in the milled powder which can probably indicate that the carbon diffused and formed phases in the consolidated sample. Moreover, the origin of this diffusion could be also the graphite molds using during the consolidation stages [14,16]. Consistent with the XRD results, the major phase identified in the microstructure should have a bcc-type structure with a lattice parameter of $0.3156 \mathrm{~nm}$ for both compositions.
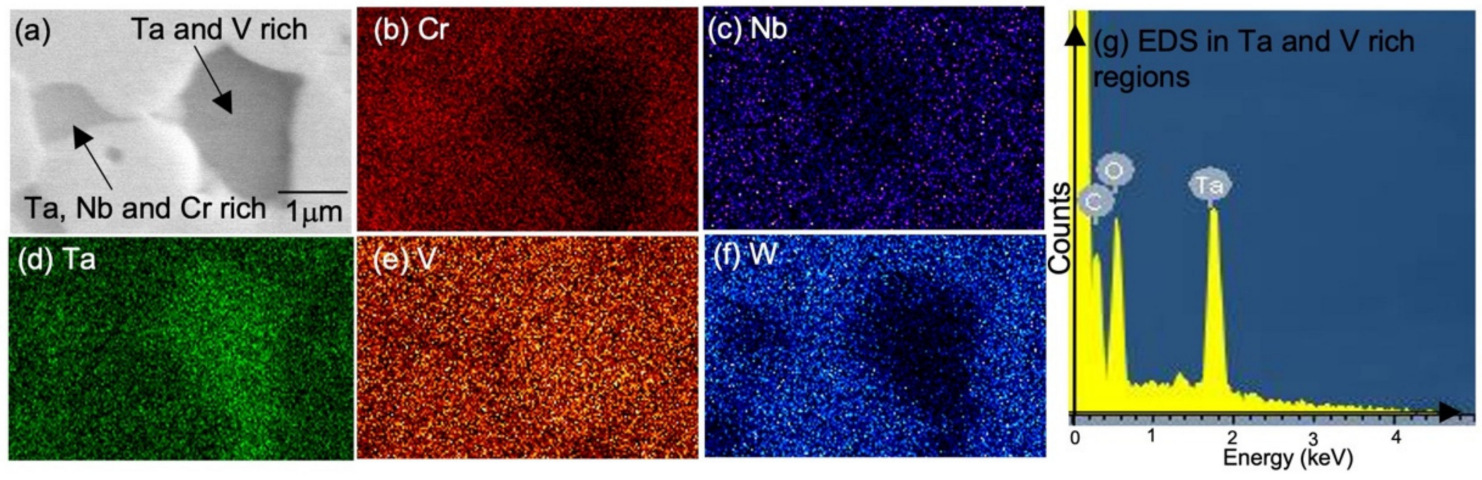

Figure 3. (a) SE image of the CrNbTaVW microstructure and the corresponding EDS maps for the (b) Cr-Lalpha, (c) Nb-Lalpha, (d) Ta-Lalpha, (e) V-Lalpha, (f) W-Lalpha and (g) EDS spectrum for regions rich in $\mathrm{Ta}$ and $\mathrm{V}$.

Table 4 shows the hardness and the elastic modulus measured for consolidated $\mathrm{CrNbTaVW}$ and $\mathrm{CrNbTaVW}_{1.7}$ high entropy alloys. The indentation hardness of the equiatomic composition was measured to be $19 \mathrm{GPa}$, whereas that of the non-equiatomic one was 17.5 GPa, this difference was also observed for the microscopic indentations (1380 and 1240, respectively). The relative small error bounds are attributed to the homogeneous microstructures. The equiatomic $\mathrm{CrNbTaVW}$ sample, as illustrated in Figure $2 \mathrm{~b}$, presented a much refined microstructure than that of $\mathrm{CrNbTaVW}_{1.7}$, which implies a size-dependent strengthening behavior described by the Hall-Petch relationship. Since $\mathrm{CrNbTaVW}$ presents a smaller grain size, the required stress to pass dislocations across grain boundaries in crystal materials increases and therefore increases hardness.

Furthermore, the exhibited hardness results for both samples are considerably higher than those reported for other HEAs with similar compositions (mostly in general in the range $140 \mathrm{HV}$ to $900 \mathrm{HV}$ ). Even though refractory metals present strong atomic bonding and hard crystal structures, HEAs are strengthened by significant lattice distortion (i.e., 
solid-solution hardening) [17]. On the other hand, the elastic modulus of the alloys obtained via nano-indentation are lower than the one for pure tungsten (400 GPa) but considerably higher than that of pure vanadium or niobium (128 GPa and $103 \mathrm{GPa}$, respectively) [18]. These results may be associated with the extensive solid solution formation between the constituents, which improves the ductility and strength of the alloys over non-alloyed metals [19].

Table 4. Hardness and Elastic modulus for both compositions $\mathrm{CrNbTaVW}_{\text {and }} \mathrm{CrNbTaVW}_{1.7}$.

\begin{tabular}{cccc}
\hline Composition & Hardness Vickers & Hardness Nano (GPa) & Elastic Modulus \\
\hline CrNbTaVW & $1380 \pm 40$ & $19.0 \pm 0.7$ & $329 \pm 7$ \\
\hline CrNbTaVW $_{1.7}$ & $1240 \pm 20$ & $17.5 \pm 0.3$ & $316 \pm 4$ \\
\hline
\end{tabular}

The results of the three-point bending test are presented in Figure $4 a, b$, with the representative loading curves at each testing temperature $(298 \mathrm{~K}, 673 \mathrm{~K}$, and $873 \mathrm{~K})$ for both compositions. Both alloys show a linear elastic behavior up to fracture in all the temperature range, which indicates that the material is brittle in the temperature chosen. However, the graphs show (Figure $4 \mathrm{a}, \mathrm{b}$ ) that the strain percentage and the stress achieved for the non-equiatomic $\mathrm{CrNbTaVW}_{1.7}$ is higher than the equiatomic $\mathrm{CrNbTaVW}$ sample. Moreover, Figure $4 \mathrm{c}$ exhibits the flexural stress as a function of the testing temperatures for the $\mathrm{CrNbTaVW}$ and $\mathrm{CrNbTaVW}_{1.7}$ compositions. At $673 \mathrm{~K}$ and $873 \mathrm{~K}$ the measured flexural strengths are higher than at $298 \mathrm{~K}$, for both compositions. This fact may be attributed to the approach of the ductile-to-brittle transition temperature typical of the bcc-type structures, such as that of tungsten, a constituent of the alloy, at $623 \mathrm{~K}$ [20], which promotes a more extensive deformation, and the superior flexural strength of the system. In addition, the results show that the flexural strength for the sample with higher amount of W (more 10 at.\%) is higher with an increase of $50 \%$. This improvement of the strength was also addressed by Wu et al. [21] and it was primarily associated with the solid solution hardening caused by the $\mathrm{W}$ incorporation. The hardening effect of $\mathrm{W}$ additions in the studied alloys can be explained in terms of the variation of the interatomic spacing with the increased number of $\mathrm{W}$ atoms, which promotes a severely distorted crystal lattice. Traditional solid solution strengthening theory [22] suggests that solute atoms with large lattice and modulus mismatch with the "solvent" could be the ideal intrinsic strengthener. Among the transitional metals, $W$ exhibits both large lattice and modulus differences from those of $\mathrm{Cr}, \mathrm{Nb}, \mathrm{V}$, and, to a minor extent, Ta and thus it leads to significant short-range bending/curving of the dislocations which consequently require elevated stress to move these dislocations.

Despite the improvement of the mechanical properties with the increasing of $\mathrm{W}$ amount, the materials are brittle in the regime studied. This lack of ductility of the refractory high entropy alloys in the low-temperature regime (below $873 \mathrm{~K}$ ) was previously addressed by Senkov et al. [23] and in fact their compressive ductility is indeed limited. Furthermore, HEAs with similar compositions, NbMoTaW and NbMoTaVW, showed only yield strength above $1873 \mathrm{~K}$ [23] and, in fact, only those refractory HEAs of the TiZrHfNbTa system showed some tensile ductility at low temperatures [24]. However, the increased strength observed at temperatures close to the expected temperature window (573-823 K estimated by T. R. Barrett in [25]) of the thermal barrier, it seems not to be enough for the expected application.

After the three-point bending tests, every fracture surface was analyzed by SEM and the corresponding micrographs are presented in Figure 5 for both samples. The $\mathrm{CrNbTaVW}$ sample exhibits a fracture surface formed at $298 \mathrm{~K}$ with both intergranular (between-grains) (Figure 5a) and transgranular (through-grain) fracture (Figure 5b), the latter observed exclusively in larger-sized particles and identified by bright crystalline cleavage facets with ridge patterns. These large particles were identified as Ta-rich phases, as observed in Figure 6, and could be indeed the origin of the fracture since its size (several microns) 
was indeed too large to strengthen the alloy by precipitation hardening. The presence of these microstructural defects, as well as the lower content of W in the HEA lattice, could be the reason why, despite presenting a more refined microstructure, the mechanical strength of this alloy is considerably lower. The transgranular fracture was less common but was also observed in samples tested at $873 \mathrm{~K}$, as some residual cleavage facets (transgranular fracture produced along the crystalline planes) were still found (Figure $5 c, d$ ). Intergranular fracture caused by grain boundary decohesion took place and was indeed observed for all temperatures.
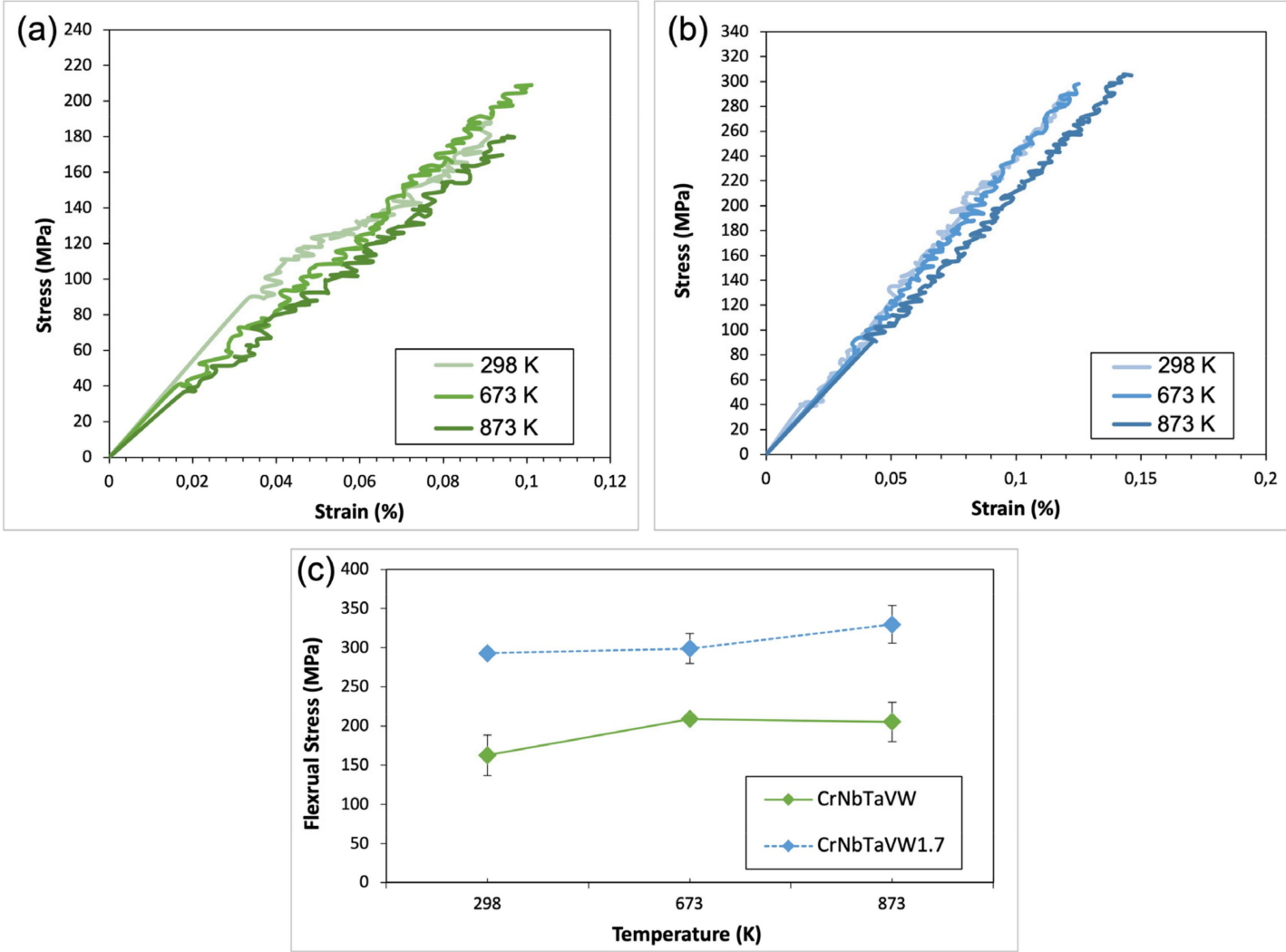

Figure 4. Stress-strain curves for (a) $\mathrm{CrNbTaVW}$ and (b) $\mathrm{CrNbTaVW}_{1.7}$ and (c) flexural strengthfracture strain of both samples at $298 \mathrm{~K}, 673 \mathrm{~K}$ and $873 \mathrm{~K}$.

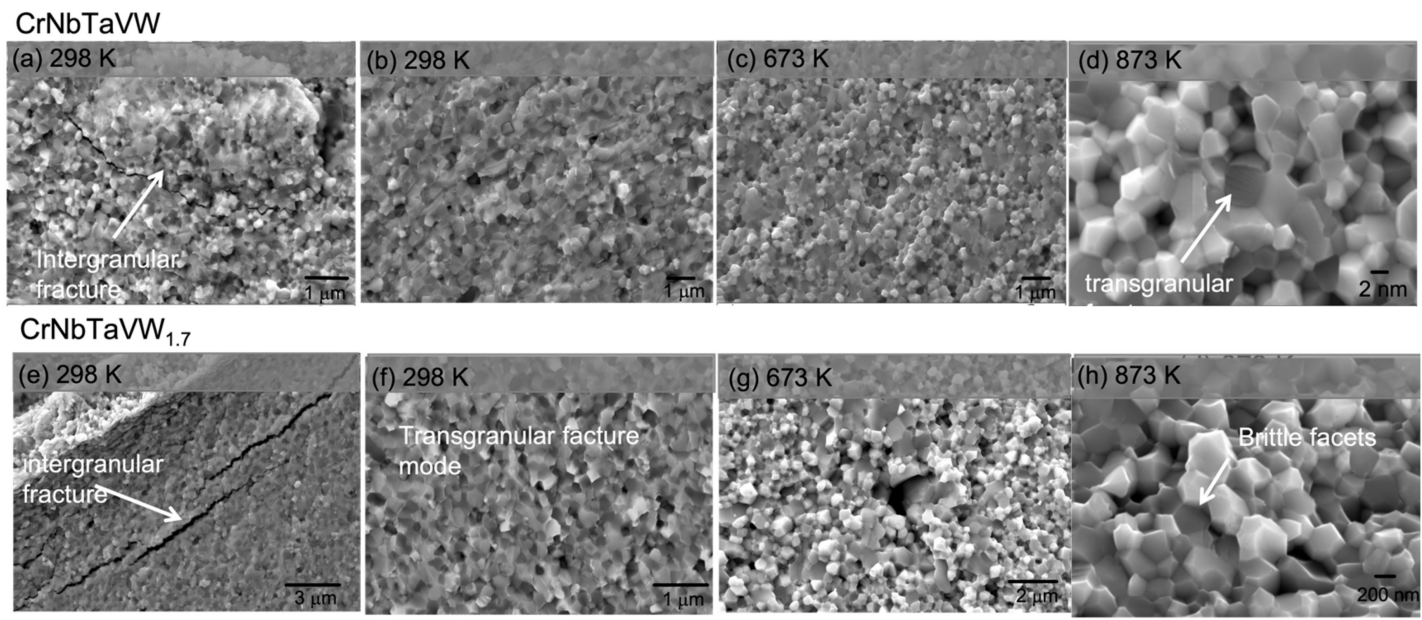

Figure 5. Types of fracture and cleavage facets observed throughout the three test temperatures for CrNbTaVW at (a) and (b) $298 \mathrm{~K}$, (c) $673 \mathrm{~K}$ and (d) $873 \mathrm{~K}$ and for $\mathrm{CrNbTaVW}_{1.7}$ at at (e) and (f) $298 \mathrm{~K}$, (g) $673 \mathrm{~K}$ and (h) $873 \mathrm{~K}$. 


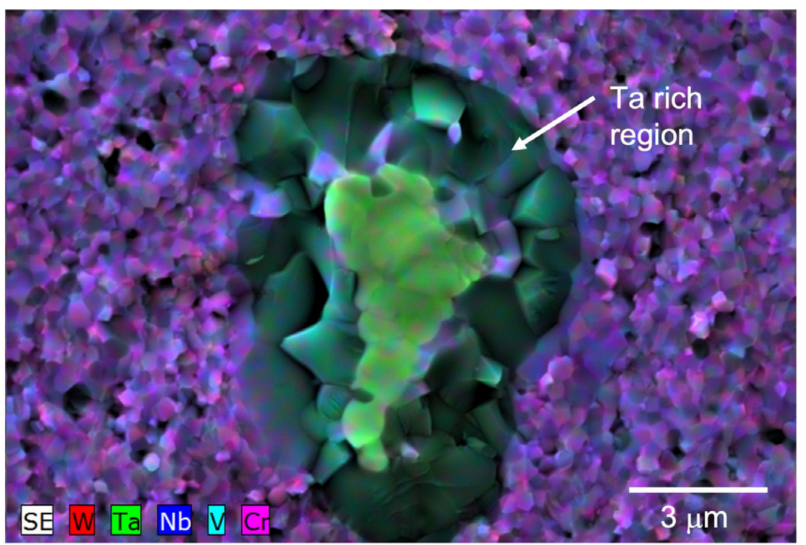

Figure 6. EDS compositional mapping of the Ta-enriched phases in the fracture surface of $\mathrm{CrNbTaVW}$ high entropy alloy.

As observed for $\mathrm{CrNbTaVW}$, three-point bending tests showed macroscopic linear elastic fracture behavior in all the temperature range, from $273 \mathrm{~K}$ to $873 \mathrm{~K}$ for the $\mathrm{CrNbTaVW}_{1.7}$ sample. On the local scale, fracture surfaces revealed a predominantly intergranular fracture at room temperature (Figure 5e). At higher temperatures, also mostly intergranular fracture modes were observed, although again, residual cleavage facets can still be observed in some grains. However, no Ta-rich large particles were detected on the fractured surfaces when compared with the equiatomic $\mathrm{CrNbTaVW}$ which point to the high mechanical strength of this alloy.

It has been reported that non-equiatomic HEA compositions present improved mechanical properties [26]. This has been ascribed to the activation of a combination of different strengthening mechanisms but a major feature is that deviating from the equiatomic composition allows avoiding brittle intermetallic phases. This approach has been used for HEAs for fusion application [16] where a reduction of hardness via increase of $W$ content translates into enhanced ductility. In the present materials there is no evidence of equiaxed dimples, associated with shear deformation and high fracture toughness, usually seen only in fcc-structure type HEA [27]. In bcc-structure type HEA cleavage provides a moderate degree of toughness but these alloys are in general more brittle than those of fcc-structure type structure. In general porosity in sintered materials is also detrimental for mechanical properties [28], the ratio of about 0.7 between macrohardness and microhardness is lower than expected with the measured densification $97 \%-95 \%$, hinting at an additional brittleness mechanism. The segregation of brittle intermetallic phases at grain boundaries have been identified as detrimental to the fracture resistance of HEAs [27]. It was demonstrated that single crystal fail by quasi-cleavage fracture with high fracture toughness even with bcc-type structure HEAs [29]. In summary, this study of $\mathrm{CrNbTaVW}$ and $\mathrm{CrNbTaVW}_{1.7}$ high entropy alloys evidences the relative brittleness of bcc-type structure HEAs, but indicates potential enhancement of mechanical behavior for such materials, via non-equiatomic compositions, enabling a lower hardness of the major phase, as well as to pinpoint general trends and thus suggest future research directions in this field.

\section{Conclusions}

In the present study, a novel $\mathrm{CrNbTaVW}$ and $\mathrm{CrNbTaVW}_{1.7}$ high entropy alloys were successfully synthesized by a combination of mechanical alloying and ultra-fast fieldassisted sintering technique. Both samples are very similar from the structural point of view, since both presented a major bcc $(a=0.316 \mathrm{~nm})$ with a multiphasic microstructure. Mechanical properties show that both materials are brittle at the temperatures studied ( $298 \mathrm{~K}, 673 \mathrm{~K}$ and $873 \mathrm{~K})$. However, an improvement in flexural strength and strain to fracture was observed in the sample with a higher amount of $W$, which is an indication of enhanced toughness due to the addition of W. As a conclusion, the characterization 
of the $\mathrm{CrNbTaVW}_{\mathrm{x}}$ high entropy alloys revealed that these materials are brittle in the temperature ranges studied, however the potential enhancement of mechanical behavior for such materials, via non-equiatomic compositions suggests an open door for future research.

Author Contributions: Conceptualization, F.A., R.M., J.B.C. and M.D.; methodology, F.A., M.D., A.P.G., E.T. and J.Y.P.; software, R.C.d.S.; validation, E.A. and M.D.; formal analysis, E.A. and M.D.; investigation, F.A. and M.D.; resources, E.A.; data curation, R.C.d.S.; writing-F.A. and M.D.; writing-review and editing, M.D.; visualization, M.D.; supervision, M.D.; project administration, M.D.; funding acquisition, E.A. All authors have read and agreed to the published version of the manuscript.

Funding: IPFN activities received financial support from "Fundação para a Ciência e Tecnologia" through projects UIDB/50010/2020 and UIDP/50010/2020. The Spanish authors would also like to acknowledge the Ministerio de Economía y Competitividad of Spain 35 (MAT2015-70780-C4-4-P and PID2019-106631GB-C44), the Comunidad de Madrid (S2018/NMT-36 4411ADITIMAT-CM) and, especially, the Universidad Politécnica de Madrid in the line of action for encouraging research from young doctors throught the project M190020074EMTG, COAT4FUSION.

Institutional Review Board Statement: Not applicable.

Informed Consent Statement: Not applicable.

Conflicts of Interest: The authors declare no conflict of interest.

\section{References}

1. Baluc, N.; Abe, K.; Boutard, J.; Chernov, V.; Diegele, E.; Jitsukawa, S.; Kimura, A.; Klueh, R.; Kohyama, A.; Kurtz, R.; et al. Status of R \& D activities on materials for fusion power reactors. Nucl. Fusion. 2007, 47, S696-S717.

2. Gumbsch, P. Brittle fracture and the brittle-to-ductile transition of tungsten. J. Nucl. Mater. 2003, 323, 304-312. [CrossRef]

3. Stork, D.; Agostini, P.; Boutard, J.; Buckthorpe, D.; Diegele, E.; Dudarev, S.; English, C.; Federici, G.; Gilbert, M.; Gonzalez, S.; et al. Developing structural, high-heat flux and plasma facing materials for a near-term DEMO fusion power plant: The EU assessment. J. Nucl. Mater. 2014, 455, 277-291. [CrossRef]

4. $\quad$ Tong, C.-J.; Chen, M.-R.; Yeh, J.-W.; Lin, S.-J.; Chen, S.-K.; Shun, T.-T.; Chang, S.Y. Mechanical performance of the Al x CoCrCuFeNi high-entropy alloy system with multiprincipal elements. Metall. Mater. Trans. A 2005, 36, 1263-1271. [CrossRef]

5. Tsai, M.-H.; Wang, C.-W.; Tsai, C.-W.; Shen, W.-J.; Yeh, J.-W.; Gan, J.-Y.; Wu, W.-W. Thermal Stability and Performance of NbSiTaTiZr High-Entropy Alloy Barrier for Copper Metallization. J. Electrochem. Soc. 2011, 158, H1161. [CrossRef]

6. Lu, C.-L.; Lu, S.-Y.; Yeh, J.-W.; Hsu, W.-K. Thermal expansion and enhanced heat transfer in high-entropy alloys. J. Appl. Cryst. 2013, 46, 736-739. [CrossRef]

7. Zhou, Y.; Lu, Z.; Zhan, M. An investigation of the erosion-corrosion characteristics of ductile cast iron. Mater. Des. 2007, 28, 260-265. [CrossRef]

8. Guo, S.; Liu, C.T. Phase stability in high entropy alloys: Formation of solid-solution phase or amorphous phase. Prog. Nat. Sci. Mater. Int. 2011, 21, 433-446. [CrossRef]

9. Otto, F.; Dlouhý, A.; Somsen, C.; Bei, H.; Eggeler, G.; George, E.P. The influences of temperature and microstructure on the tensile properties of a CoCrFeMnNi high-entropy alloy. Acta Mater. 2013, 61, 5743-5755. [CrossRef]

10. Senkov, O.N.; Woodward, C.; Miracle, D.B. Microstructure and Properties of Aluminum-Containing Refractory High-Entropy Alloys. Jom 2014, 66, 2030-2042. [CrossRef]

11. Senkov, O.N.; Miracle, D.B.; Chaput, K.J.; Couzinie, J.-P. Development and exploration of refractory high entropy alloys-A review. J. Mater. Res. 2018, 33, 3092-3128. [CrossRef]

12. The International Centre for Diffraction Data-ICDD. Available online: http://www.icdd.com/ (accessed on 23 April 2020).

13. BAM Berlin PowderCell. Available online: http://www.ccp14.ac.uk/ccp/web-mirrors/powdcell/a_v/v_1/powder/e_cell.html (accessed on 23 April 2020).

14. Zhang, Y.; Zhou, Y.J.; Lin, J.P.; Chen, G.L.; Liaw, P.K. Solid-solution phase formation rules for multi-component alloys. Adv. Eng. Mater. 2008, 10, 534-538. [CrossRef]

15. Oliver, W.C.; Pharr, G.M. An improved technique for determining hardness and elastic modulus using load and displacement sensing indentation experiments. J. Mater. Res. 1992, 7, 1564-1583. [CrossRef]

16. Waseem, O.A.; Ryu, H.J. Powder Metallurgy Processing of a $\mathrm{W}_{\mathrm{x}} \mathrm{TaTiVCr}$ High-Entropy Alloy and Its Derivative Alloys for Fusion Material Applications. Sci. Rep. 2017, 7, 1926. [CrossRef] [PubMed]

17. Kim, H.; Nam, S.; Roh, A.; Son, M.; Ham, M.-H.; Kim, J.-H.; Choi, H. Mechanical and electrical properties of NbMoTaW refractory high-entropy alloy thin films. Int. J. Refract. Met. Hard Mater. 2019, 80, 286-291. [CrossRef]

18. ASMIH Committee. ASM Handbook: Properties and Selection; ASM International: Almere, The Netherlands, 1990. 
19. Senkov, O.N.; Scott, J.M.; Senkova, S.V.; Miracle, D.B.; Woodward, C.F. Microstructure and room temperature properties of a high-entropy TaNbHfZrTi alloy. J. Alloys Compd. 2011, 509, 6043-6048. [CrossRef]

20. Stephens, J.R. Review of Deformation Behavior of Tungsten At Temperatures Less Than 0.2 Absolute Melting Temperature. In Nasa Tm X-2482; National Aeronautics and Space Administration: Washington, DC, USA, 1972.

21. Wu, Z.; Guo, W.; Jin, K.; Poplawsky, J.D.; Gao, Y.; Bei, H. Enhanced Strength and Ductility of a Tungsten-doped CoCrNi Medium Entropy Alloy. J. Mater. Res. 2018, 33, 3301-3309. [CrossRef]

22. Rate Processes in Plastic Deformation of Materials: Proceedings from the Google Books. Available online: https: //books.google.pt/books/about/Rate_Processes_in_Plastic_Deformation_of.html?id=tXsMYAAACAAJ\&redir_esc=y (accessed on 3 January 2022).

23. Senkov, O.N.; Wilks, G.B.; Scott, J.M.; Miracle, D.B. Mechanical properties of Nb25Mo25Ta25W25 and V20Nb20Mo20Ta20W20 refractory high entropy alloys. Intermetallics 2011, 19, 698-706. [CrossRef]

24. Senkov, O.N.; Pilchak, A.L.; Semiatin, S.L. Effect of Cold Deformation and Annealing on the Microstructure and Tensile Properties of a HfNbTaTiZr Refractory High Entropy Alloy. Metall. Mater. Trans. A 2018, 49, 2876-2892. [CrossRef]

25. Barrett, T.; McIntosh, S.; Fursdon, M.; Hancock, D.; Timmis, W.; Coleman, M.; Rieth, M.; Reiser, J. Enhancing the DEMO divertor target by interlayer engineering. Fusion Eng. Des. 2015, 98-99, 1216-1220. [CrossRef]

26. Li, D.; Raabe, Z. Strong and Ductile Non-equiatomic High-Entropy Alloys 2017.pdf. J. Met. 2017, 69, $2099-2106$.

27. Li, W.; Liaw, P.K.; Gao, Y. Fracture resistance of high entropy alloys: A review. Intermetallics 2018, 99, 69-83. [CrossRef]

28. Ternero, F.; Rosa, L.G.; Urban, P.; Montes, J.M.; Cuevas, F.G. Influence of the total porosity on the properties of sintered materials-A review. Metals 2021, 11, 730. [CrossRef]

29. Zou, Y.; Okle, P.; Yu, H.; Sumigawa, T.; Kitamura, T.; Maiti, S.; Steurer, W.; Spolenak, R. Fracture properties of a refractory high-entropy alloy: In situ micro-cantilever and atom probe tomography studies. Scr. Mater. 2017, 128, 95-99. [CrossRef] 